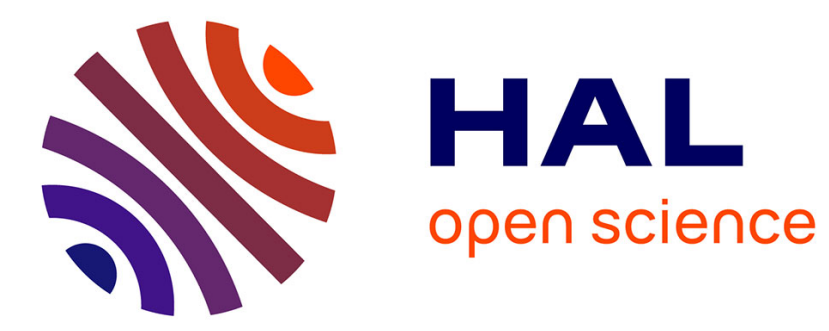

\title{
Tracking marine mammals in 3D using electronic tag data
}

\author{
Christophe Laplanche, Tiago Marques, Len Thomas
}

\section{To cite this version:}

Christophe Laplanche, Tiago Marques, Len Thomas. Tracking marine mammals in 3D using electronic tag data. Methods in Ecology and Evolution, 2015, vol. 6 ( $\mathrm{n}^{\circ}$ 9), pp. 987-996. 10.1111/2041210X.12373 . hal-01227976

\section{HAL Id: hal-01227976 https://hal.science/hal-01227976}

Submitted on 12 Nov 2015

HAL is a multi-disciplinary open access archive for the deposit and dissemination of scientific research documents, whether they are published or not. The documents may come from teaching and research institutions in France or abroad, or from public or private research centers.
L'archive ouverte pluridisciplinaire HAL, est destinée au dépôt et à la diffusion de documents scientifiques de niveau recherche, publiés ou non, émanant des établissements d'enseignement et de recherche français ou étrangers, des laboratoires publics ou privés. 


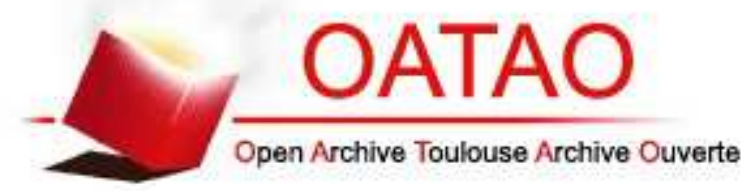

\section{Open Archive TOULOUSE Archive Ouverte (OATAO)}

OATAO is an open access repository that collects the work of Toulouse researchers and makes it freely available over the web where possible.

This is an author-deposited version published in : http://oatao.univ-toulouse.fr/ Eprints ID : 14435

To link to this article : doi: 10.1111/2041-210X.12373

URL : http://dx.doi.org/10.1111/2041-210X.12373

To cite this version : Laplanche, Christophe and Marques, Tiago and Thomas, Len Tracking marine mammals in $3 D$ using electronic tag data. (2015) Methods in Ecology and Evolution, vol. 6 (n 9). pp. 987996. ISSN 2041-210X

Any correspondance concerning this service should be sent to the repository administrator: staff-oatao@ listes-diff.inp-toulouse.fr 


\title{
Tracking marine mammals in 3D using electronic tag data
}

\author{
Christophe Laplanche $^{1,2}$, Tiago A. Marques ${ }^{3 *}$ and Len Thomas ${ }^{3}$ \\ ${ }^{1}$ Université de Toulouse, INP, UPS, EcoLab (Laboratoire Ecologie Fonctionnelle et Environnement), ENSAT, Avenue de \\ I'Agrobiopole, 31326 Castanet Tolosan, France; ${ }^{2}$ ECOLAB, CNRS, 31326 Castanet Tolosan, France; and ${ }^{3}$ Centre for \\ Research into Ecological and Environmental Modelling, The Observatory, Buchanan Gardens, University of St Andrews, \\ St Andrews, KY16 9LZ Scotland, UK
}

\section{Summary}

1. Information about at-depth behaviour of marine mammals is fundamental yet very hard to obtain from direct visual observation. Animal-borne multisensor electronic tags provide a unique window of observation into such behaviours.

2. Electronic tag sensors allow the estimation of the animal's 3-dimensional (3D) orientation, depth and speed. Using tag flow noise level to provide an estimate of animal speed, we extend existing approaches of 3D track reconstruction by allowing the direction of movement to differ from that of the animal's longitudinal axis.

3. Data are processed by a hierarchical Bayesian model that allows processing of multisource data, accounting for measurement errors and testing hypotheses about animal movement by comparing models.

4. We illustrate the approach by reconstructing the 3D track of a 52-min deep dive of a Blainville's beaked whale Mesoplodon densirostris adult male fit with a digital tag (DTAG) in the Bahamas. At depth, the whale alternated regular movements at large speed $\left(>1.5 \mathrm{~m} \mathrm{~s}^{-1}\right)$ and more complex movements at lower speed $\left(<1.5 \mathrm{~m} \mathrm{~s}^{-1}\right)$ with differences between movement and longitudinal axis directions of up to $28^{\circ}$. The reconstructed $3 \mathrm{D}$ track agrees closely with independent acoustic-based localizations.

5. The approach is potentially applicable to study the underwater behaviour (e.g. response to anthropogenic disturbances) of a wide variety of species of marine mammals fitted with triaxial magnetometer and accelerometer tags.

Key-words: animal movement modelling, dead reckoning, electronic tag, flow noise, hierarchical Bayesian modelling, track reconstruction, triaxial magnetometer and accelerometer

\section{Introduction}

The use of animal-borne autonomous recording tags to collect information for inferences on movement, ecology, physiology and behaviour is becoming widespread, providing an unprecedented window into these biological processes and leading to otherwise unattainable discoveries, especially at sea where animal behaviour is hard to observe directly (Ropert-Coudert \& Wilson 2005; Bograd et al. 2010).

Initially used simply to identify animals, over time tags became equipped with thermometers and barometers, followed by accelerometers, magnetometers, gyroscopes, microphones, hydrophones, GPSs, and even video (Marshall et al. 2007; Burgess 2009; Johnson, Aguilar de Soto \& Madsen 2009; Rutz \& Troscianko 2013). Some tags provide direct information on location, while others do not. For those that do, say via GPS or radio tracking, a common approach has been to use state space models or hidden Markov models to reconstruct two-dimensional tracks (Jonsen et al. 2012; Beyer et al. 2013; Langrock et al. 2014). However, most marine mammals spend a large proportion of their time at depth; hence, accounting for the depth component might be funda-

*Correspondence author. E-mail: tam2@st-andrews.ac.uk mental, depending on each study's objectives (Tracey et al. 2014).

Published tracks in 3 dimensions (3D) are based on some form of dead reckoning (Wilson et al. 2007): each position is predicted by updating the previous time step position considering an estimate of the animal's current direction and speed. One option is to infer animal 3D speed from 3D orientation (computed from accelerometer and magnetometer data) and vertical speed (from depthmeter data). However, this is sensitive to error in depth measurements, notably when animal movement is close to horizontal. This has led to estimating speed from other sources than depthmeters, namely tag flow noise (Simon et al. 2009; Ware, Friedlaender \& Nowacek 2011). All such methods have required the assumption that the direction of animal movement coincides with the direction of its longitudinal (rostro-caudal for a whale) axis; that is, the animal moves towards where it is pointing. If this does not hold, bias can be expected, and the resulting track will be unreliable (Johnson, Aguilar de Soto \& Madsen 2009). Further, errors accumulate over time, a phenomenon referred to as drift (Wilson et al. 2007). Additional drifting due to external factors can occur (Shiomi et al. 2008). Therefore, while tags are very useful to establish relative positions of animals, inferring absolute position is questionable with existing procedures: the term 
pseudo-track is used to reinforce the notion that absolute position is unknown (Hazen et al. 2009). Also for this reason, dead-reckoning tracks are often 'anchored' to known positions (Zimmer et al. 2005; Friedlaender et al. 2009; Hazen et al. 2009). These are sometimes referred to as georeferenced tracks, to convey the notion of absolute position on the earth sphere. However, measurement error in positions is typically ignored, and the way the pseudo-track is combined with these is not explicitly described (Davis et al. 2001; Mitani et al. 2003; Tyson et al. 2012). Nonetheless, implementation details can have considerable impact on the estimated track, as well as (if estimated) on its precision.

We consider digital tags (DTAGs) (Johnson \& Tyack 2003) as an example. DTAGs include triaxial accelerometer and magnetometer sensors, a pressure sensor (sampling rate up to $50 \mathrm{~Hz}$ ) and two hydrophones (up to $192 \mathrm{kHz}$ ) (Johnson \& Tyack 2003). Other tags (e.g. 'OpenTag'; Loggerhead Instruments, Sarasota, FL, USA) include triaxial magnetometers and accelerometers. Around 20 marine mammal species (>1000 deployments) including whales, dolphins and pinnipeds have been fitted with DTAGs (M. Johnson, pers. comm.). Such tags have become widespread in marine mammal studies, allowing inferences about at-depth behaviour and ecophysiology (Watwood et al. 2006; Shaffer et al. 2013). DTAGs were originally developed to infer behaviour and relative movement rather than absolute location, having been used extensively for this purpose - for example recent work on feeding behaviour in baleen whales (Simon, Johnson \& Madsen 2012; Ware et al. 2014 and references therein). However, DTAG data have been used to reconstruct 3D dives of animals (Davis et al. 2001; Johnson \& Tyack 2003; Mitani et al. 2003; Madsen et al. 2005). Bespoke software is now available to process tag data into tracks [the R packages animalTrack, Farrell \& Fuiman (2013), and TrackReconstruction, Battaile (2014), and to depict 3D tracks Trackplot, Ware et al. (2006)]. An estimated position without an associated measure of uncertainty can be misleading, providing overconfidence in the reported estimate. Nonetheless, existing software does not provide uncertainty on position estimates, so these are never reported.

Extending dead reckoning and georeferencing methods described earlier, we develop a new way to use magnetometer and accelerometer tag data to reconstruct 3D tracks and estimate associated uncertainty. We explicitly (i) incorporate measurement error, both from the tag and from estimated positions, in the input data and propagate this error through to the estimated track; (ii) include information about animal speed both from change in depth given orientation and from tag flow noise; and (iii) utilize the additional information from both sources of speed information to relax the assumption that the animal moves in the direction it is pointed. Our model is superficially similar to well-known 2D random walk models by, for example, Jonsen, Flemming \& Myers (2005), Morales et al. (2004) and McClintock et al. (2012) in that, like them, we model animal speed (i.e. step length) and movement direction in discrete time and continuous space and use Bayesian methods to link models to data. However, assumptions about animal movement differ. Random walk models make distributional assumptions about step length and direction (or turning angle); hence, resulting track estimates are a combination of the assumed movement model and the input data (filtered through the observation process); by contrast, we do not make such assumptions; hence, our estimated tracks are a function of the data and observation process alone. In this sense, our approach is more 'data focused', but is also more reliant on having high-frequency, high-quality data to produce a realistic track. We return to these issues in the Discussion.

We illustrate our method by reconstructing a 52-min dive of a tagged Blainville's beaked whale Mesoplodon densirostris (Laplanche, Marques \& Thomas 2015), for which independent underwater localizations are available. These are not used in model fitting; instead, we use them to evaluate the accuracy of the estimated track derived from tag data alone. Finally, we discuss the capabilities of the approach and possible improvements.

\section{Materials and methods}

\section{TAG MEASUREMENTS AND COORDINATE SYSTEMS}

We consider three coordinate systems (or frames) to accurately describe animal movement and tag data: (i) the Earth frame, a cartographic projected coordinate system ( $x$-axis south-north, positive north; $y$-axis east-west, positive west; $z$-axis bottom-up, positive up; origin is some arbitrary location at the sea surface), (ii) the animal frame ( $x$-axis, longitudinal axis, positive forward; $y$-axis, right-left axis, positive left; $z$ axis, dorso-ventral axis, positive up; origin is the geometric centre of the animal), and (iii) the tag frame ( $x$-, $y$-, $z$-axes are internally defined; origin is the centre of the tag) - this latter frame is required because the tag is not always placed with the same orientation on the animal.

An animal's 3D track is the time-series of its 3D location; more specifically the 3D Cartesian coordinates of the origin of the animal frame in the Earth frame denoted $\boldsymbol{x}(t)=(x(t), y(t), z(t))$ at time $t$. Animal 3D speed is the time derivative of $\boldsymbol{x}(t)$; the speed of translation of the animal frame in the Earth frame denoted $\boldsymbol{v}(t)=\left(v_{x}(t), v_{y}(t), v_{z}(t)\right)$. The orientation of a $3 \mathrm{D}$ object in space is unambiguously described in terms of heading $h$ (rotation to the $z$-axis, $\left.h \in\left(-180^{\circ}, 180^{\circ}\right]\right)$, pitch $p$ ( $y$-axis, $\left.p \in\left(-90^{\circ}, 90^{\circ}\right]\right)$ and roll $r$ ( $x$-axis, $\left.r \in\left(-180^{\circ}, 180^{\circ}\right]\right)$ with respect to some frame of reference. The animal's 3D orientation at time $t$ is represented by its heading $h(t)$ (positive eastwards), pitch $p(t)$ (positive upwards) and roll $r(t)$ (positive rightwards), with respect to the Earth frame. Tag data are not directly available in the Earth frame. Accelerometer and magnetometer measure the Earth's gravitational and magnetic fields in the tag frame. The conversion of Earth's gravitational and magnetic fields between animal and Earth frames is achieved via rotation matrices described in the next section. The conversion of raw accelerometer and magnetometer data in the tag frame into the animal frame is achieved in a similar way. Description of the latter process, together with the processing of acoustic data into flow noise level, is deferred to Section 'Example data set'.

\section{THE STATISTICAL MODEL}

We describe the full statistical model here. Approximations used in practice for computational efficiency are described in Section 'Bayesian computation and approximating model'. 
The objective is to use available tag data (Earth's gravitational and magnetic fields in the animal frame, depth, flow noise level), and independent positional data, if available, to infer unknown, latent variables characterizing animal movement $(\boldsymbol{x}(t), \boldsymbol{v}(t), h(t), p(t)$ and $r(t))$. Our implementation utilizes a hierarchical Bayesian model (HBM). The overall model structure is illustrated in Fig. 1, relating latent and measured variables as detailed below. For clarity, the model is presented in four sections: (i) estimation of animal orientation from accelerometer, magnetometer and depthmeter measurements; (ii) estimation of speed from flow noise measurement and direction of movement from a combination of speed, orientation and change in depth; (iii) track estimation; and (iv) incorporation of independent positional information.

We define $t_{0}$ and $t_{\text {end }}$ as the track start and end times, $t \in\left[t_{0}, t_{\text {end }}\right]$.

\section{Animal 3D orientation}

The expected values $\boldsymbol{A}^{a}(t)$ and $\boldsymbol{M}^{\boldsymbol{a}}(t)$ of the 3D Earth gravitational and magnetic fields in the animal frame (superscript $a$ ) at time $t$ are

$$
\begin{aligned}
\boldsymbol{A}^{a}(t) & =T(t) \boldsymbol{A}^{e} \\
\boldsymbol{M}^{a}(t) & =T(t) \boldsymbol{M}^{e},
\end{aligned}
$$

where $T(t)$ is a rotation matrix that switches from the Earth frame to the animal frame given by

$$
\begin{aligned}
T(t)= & \left(\begin{array}{ccc}
1 & 0 & 0 \\
0 & \cos r(t) & \sin r(t) \\
0 & -\sin r(t) & \cos r(t)
\end{array}\right) \\
& \times\left(\begin{array}{ccc}
\cos p(t) & 0 & \sin p(t) \\
0 & 1 & 0 \\
-\sin p(t) & 0 & \cos p(t)
\end{array}\right) \\
& \times\left(\begin{array}{ccc}
\cosh (t) & \sin h(t) & 0 \\
-\sin h(t) & \cos h(t) & 0 \\
0 & 0 & 1
\end{array}\right),
\end{aligned}
$$

and $\boldsymbol{A}^{e}$ and $\boldsymbol{M}^{e}$ are the values of the 3D Earth gravitational and magnetic fields in the Earth frame (superscript $e$ ) at the tagging location and time. Given the relative small scale of most studies, ours included, compared to these 3D Earth fields, these can safely be treated as constants. They can be either measured or derived from models of the gravitational and Earth magnetic fields.
Measured (superscript obs) values of the Earth gravitational $\left(\boldsymbol{A}^{\boldsymbol{a}, \boldsymbol{o b s}}(t)\right)$ and magnetic fields $\left(\boldsymbol{M}^{\boldsymbol{a}, \boldsymbol{o b s}}(t)\right)$ in the animal frame at time $t$ are modelled as multivariate Gaussian distributions (MVN)

$$
\begin{aligned}
\boldsymbol{A}^{\boldsymbol{a}, \boldsymbol{o b s}}(t) & \sim \operatorname{MVN}\left(\boldsymbol{A}^{\boldsymbol{a}}(t), \boldsymbol{\Sigma}_{\boldsymbol{A}}(t)\right) \\
\boldsymbol{M}^{\boldsymbol{a}, \boldsymbol{o b s}}(t) & \sim \operatorname{MVN}\left(\boldsymbol{M}^{\boldsymbol{a}}(t), \boldsymbol{\Sigma}_{\boldsymbol{M}}(t)\right)
\end{aligned}
$$

where $\boldsymbol{\Sigma}_{\boldsymbol{A}}(t)$ and $\boldsymbol{\Sigma}_{\boldsymbol{M}}(t)$ are time-dependent covariance matrices (see Appendix S1 for details). The observed animal depth is

$z^{o b s}(t) \sim \operatorname{Normal}\left(z(t), \sigma_{z}^{2}\right), z^{o b s}(t)<=0$,

where $z(t)$ is the unobserved true depth of the animal in the Earth frame and $\sigma_{z}^{2}$ is the depthmeter measurement error variance.

\section{Animal speed and direction of movement}

We explicitly relax what we refer in the following as the equal pitch assumption: that the direction of animal movement coincides with the direction of its longitudinal axis. Animal speed animal at time $t$ is

$\left\{\begin{array}{l}v_{x}(t)=\cos h^{\prime}(t) \cos p^{\prime}(t) v(t) \\ v_{y}(t)=-\sin h^{\prime}(t) \cos p^{\prime}(t) v(t) \\ v_{z}(t)=\sin p^{\prime}(t) v(t)\end{array}\right.$

where $v(t)=\|\boldsymbol{v}(t)\|, h^{\prime}(t)$ and $p^{\prime}(t)$ are the Euclidean norm, the heading (positive eastwards) and the pitch (positive upwards) in the Earth frame of the speed vector of the animal at time $t$. Differences of orientations of the longitudinal axis and the speed vector are modelled as differences in respective pitch angles

$p^{\prime}(t) \sim \operatorname{Normal}\left(p(t), \sigma_{p}^{2}\right), \quad p^{\prime}(t) \in(-90,90]$,

(eqn 6)

where $\sigma_{p}^{2}$ is the variance of the pitch difference $\Delta p(t)=p(t)-p^{\prime}(t)$. We refer in the following to this as the unequal pitch assumption and to $\Delta p$ $(t)$ as pitch anomaly. A positive pitch anomaly occurs when the animal points its longitudinal axis higher than expected by its swimming direction, and vice versa (Fig. 2). Pitch anomaly can be the result of a pitch and/or a heading movement in the animal frame depending on the roll. For reasons discussed later, we do not consider heading anomaly, hence assuming $h(t)=h^{\prime}(t)$.

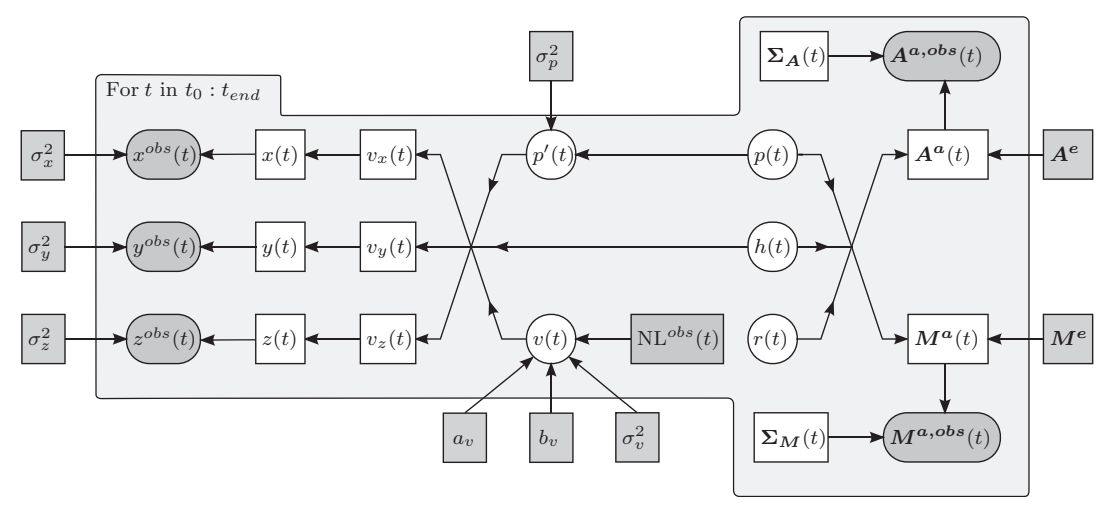

Fig. 1. Directed acyclic graph (DAG) illustrating the relationship between model parameters and measured variables. Measured variables (in dark grey) are either modelled as random variables (circles and rounded rectangles) or are considered as known (rectangles). Parameters (in white) are either defined by a stochastic formula (circles and rounded rectangles) or are deterministic resultants of upstream nodes (rectangles). Variables indexed with $t$ are time dependent (grey polygon). The 3D orientation of the animal $(h(t), p(t), r(t))$ is estimated from the accelerometer and magnetometer $\left(\boldsymbol{A}^{\boldsymbol{a}, \boldsymbol{o b s}}(t), \boldsymbol{M}^{\boldsymbol{a}, \boldsymbol{o b s}}(t)\right)$ data. The 3D orientation and norm $\left(h(t), p^{\prime}(t), v(t)\right)$ of the animal speed vector is used to compute the 3D speed vector $\left(v_{x}(t), v_{y}(t), v_{z}(t)\right)$ and resulting track $(x(t), y(t), z(t))$. The model allows for the possibility that the animal has a swimming direction $\left(p^{\prime}(t)\right)$ that is distinct from, yet statistically related to, the $3 \mathrm{D}$ orientation of its body $(p(t))$. 


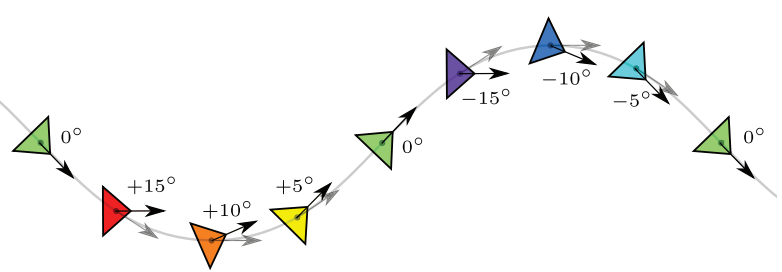

Fig. 2. Pitch anomaly $\Delta p(t)=p(t)-p^{\prime}(t)$ is the difference between the pitch $(p(t))$ of the orientation of the animal's longitudinal axis (black arrows) and the pitch $\left(p^{\prime}(t)\right)$ of the animal's speed vector (grey arrows). A positive pitch anomaly highlights movements when the animal points its longitudinal axis higher than expected by its swimming direction, and vice versa. The 3D whale track (grey line) and vectors are projected on a vertical plane. The colour legend for pitch anomaly is the same as what is used in Fig. 4 (green: no anomaly; from yellow to red: increasing positive anomaly; from cyan to violet: decreasing negative anomaly), and angles between pairs of arrows have been inflated in the current plot for the ease of representation.

Animal speed is related to background noise level $\mathrm{NL}(t)$ at time $t$ assuming

$v(t) \sim \operatorname{Normal}\left(a_{v}+b_{v} \log _{10}(\mathrm{NL}(t)), \sigma_{v}^{2}\right), v(t) \geq 0$,

where $a_{v}$ and $b_{v}$ are regression parameters and $\sigma_{v}$ is the residual standard error (Appendix S2).

\section{Animal 3D track}

Animal Cartesian coordinates at time $t+\Delta t$ are computed from coordinates at time $t$ and speed:

$\left\{\begin{array}{l}x(t+\Delta t)=x(t)+v_{x}(t) \Delta t \\ y(t+\Delta t)=y(t)+v_{y}(t) \Delta t \\ z(t+\Delta t)=z(t)+v_{z}(t) \Delta t\end{array}\right.$

\section{Independent positional information}

In our application, we only use information about the dive starting position, assumed to have been observed with known error. We model this as

$$
\left\{\begin{array}{l}
x^{o b s}\left(t_{0}\right) \sim \operatorname{Normal}\left(x\left(t_{0}\right), \sigma_{x}^{2}\left(t_{0}\right)\right) \\
y^{\text {obs }}\left(t_{0}\right) \sim \operatorname{Normal}\left(y\left(t_{0}\right), \sigma_{y}^{2}\left(t_{0}\right)\right)
\end{array}\right.
$$

where $\sigma_{x}^{2}\left(t_{0}\right)$ and $\sigma_{y}^{2}\left(t_{0}\right)$ are known variance terms. If the absolute start position is unknown, arbitrary values are provided for $\left(x^{o b s}\left(t_{0}\right), y^{o b s}\left(t_{0}\right)\right)$ with null variances $\left(\sigma_{x}^{2}\left(t_{0}\right)=\sigma_{y}^{2}\left(t_{0}\right)=0\right)$; estimated locations become relative to this position.

Similarly, additional animal positions might be used to improve the track reconstruction process. When at the surface, these could come from visual observations, animal-borne GPS or satellite receivers. When underwater, these could come from passive (or active) acoustic localizations.

\section{Priors}

Prior distributions are required on all top-level random variables in the hierarchical model. Observation variance parameters are assumed known, hence not requiring priors. We also assume the relationship between measured noise level and speed is known with certainty (see Section 'Bayesian computation and approximating model' and Discus- sion). These variables are shown as grey boxes in Fig. 1. The remaining top-level variables are pitch, heading and roll at each time step, for which uniform distributions are assumed:

$\left\{\begin{array}{l}p(t) \sim \operatorname{Uniform}(-90,90) \\ h(t) \sim \operatorname{Uniform}(-180,180) \\ r(t) \sim \operatorname{Uniform}(-180,180)\end{array}\right.$

\section{BAYESIAN COMPUTATION AND APPROXIMATING MODEL}

The model described by eqns (1-10) is not analytically tractable; however, samples from the posterior distribution of latent variables can be simulated via Markov chain Monte Carlo (MCMC). For this, we used OpenBUGS version 3.2.1, open-source version of WinBUGS (Ntzoufras 2009). BUGS code is available as Appendix S3. Tag data pre-processing and output post-processing were implemented in $\mathrm{R}$ ( $\mathrm{R}$ Core Team 2013).

Initial runs showed that the full model was highly computer-intensive. Two procedures were implemented to reduce computing time, both of which mean we fit an approximation to the full model. First, the model was divided into three stages (and each stage was analysed in turn): (i) compute animal 3D orientation (eqns $1-4,10$ ); (ii) calibrate the speed-noise relationship (eqn 7); and (iii) compute animal 3D track (eqns $5,6,8,9$ ). Uncertainty was propagated across stages by modelling stage outputs as Gaussians, with mean and variance equal to the corresponding posterior values, using this distribution as input to the next stage. However, in moving from stage (ii) to (iii), the parameters of the speed-noise model were assumed known. Secondly, in computing stages (i) and (iii), the track was divided into 1-min pieces. Each piece was run in parallel using a high-performance computing resource (HPR). Pieces were then joined and uncertainty from the end of each piece propagated to the beginning of the next (see Appendix S4 for details and discussion for possible impacts).

Markov chain Monte Carlo convergence was assessed by computing the interchain variances of the simulated latent variable samples across four chains. For each chain, once convergence was reached, 10000 samples were simulated; these were thinned to 1000 independent samples per chain, with thinning guided by analysing the autocorrelation function of the posterior samples. Reported point estimates are posterior means, standard errors are posterior standard deviations (reported as mean \pm standard error), and reported interval estimates are $2.5 \%$ and $97.5 \%$ posterior marginal quantile estimates.

\section{ALTERNATIVE MODELS FOR PITCH ANOMALY}

The model assumes a fixed pitch anomaly standard deviation $\sigma_{p}$ (see Discussion for a relaxation of this assumption). To investigate how pitch anomaly varied along the track, we repeated the above analysis considering three different values for $\sigma_{p}: 0^{\circ}, 5^{\circ}$ and $10^{\circ}$. These represent three different models and we denote them $\mathcal{M}_{0}, \mathcal{M}_{5}$ and $\mathcal{M}_{10}$, respectively.

Models were compared, for each track piece, using the deviance information criterion (DIC; Spiegelhalter et al., 2002), a goodness-offit index penalized for model complexity, similar in spirit to Akaike's information criterion; smaller values are considered better (see Section 4 for a discussion of alternative model selection measures). Following Gelman et al. (2003), we estimated model complexity as $p_{v}=\operatorname{var}\{-2 \log [p(\theta \mid y)]\} / 2$. The models do not share the same complexity: $\mathcal{M}_{0}$ is the least complex $\left(p^{\prime}(t)\right.$ is perfectly known given $\left.p(t)\right)$, which is less complex than $\mathcal{M}_{5}\left(p^{\prime}(t)\right.$ estimated under the more relaxed 
constraint of eqn (6) with $\sigma_{p}=5^{\circ}$ ) which is itself less complex than $\mathcal{M}_{10}$ (even more relaxed constraint with $\sigma_{p}=10^{\circ}$ ). In the Results, we report which model was favoured in each minute of the track.

\section{EXAMPLE DATA SET}

For illustration, we used a Mesoplodon densirostris Blainville's beaked whale adult male tagged on the 5th September 2007 (tag on position: $24.3839 \mathrm{~N}, 77.5615 \mathrm{~W}$ ) at AUTEC (Atlantic Undersea Test and Evaluation Center, an instrumented US Navy testing range in the Bahamas). AUTEC details and a different analysis of this DTAG data can be found in Ward et al. (2011). We illustrate the methods using the first deep dive, which lasted 51'20" (full tag deployment: $16 \mathrm{~h}$, five deep dives). Mesoplodon densirostris depth profiles have been modelled using behaviour states (Langrock et al. 2013), and deep dives can be divided into descent, foraging and ascent phases: here, the whale fluked up and initiated its dive at arbitrarily fixed $t_{0}=0$, ended its descent and started active searching for prey at $t_{B}=7^{\prime} 50^{\prime \prime}$, stopped active searching for prey and initiated its ascent at $t_{C}=35^{\prime} 30^{\prime \prime}$, and reached the surface at $t_{\text {end }}=51^{\prime} 20^{\prime \prime}$.

The magnetic field was computed by using the IGRF11 (11th Generation International Geomagnetic Reference Field) Earth's main magnetic field model (International Association of Geomagnetism and Aeronomy, Working Group V-MOD, 2010). The magnetic field at the tagging location and time was $\boldsymbol{M}^{e}=(25736,3205,-35522) \mathrm{nT}$ (declination: $7 \cdot 15^{\circ} \mathrm{W}$; inclination: $54.08^{\circ}$ down). The gravitational field was $\boldsymbol{A}^{e}=(0,0,-9.79) \mathrm{m} \mathrm{s}^{-2}$. Arbitrary null values were provided for the location of the whale at the beginning of the dive $\left(x^{o b s}\left(t_{0}\right)=y^{o b s}\left(t_{0}\right)=0 \mathrm{~m}\right.$ with $\left.\sigma_{x}^{2}\left(t_{0}\right)=\sigma_{y}^{2}\left(t_{0}\right)=0 \mathrm{~m}\right)$.

Raw tag-frame accelerometer and magnetometer data were converted into animal-frame accelerometer and magnetometer data as described by Johnson \& Tyack (2003). Accelerometer, magnetometer and depthmeter data were low-pass filtered by using a 1-second, squared-window rolling mean before being downsampled at $1 \mathrm{~Hz}$ $(\Delta t=1 \mathrm{~s})$. Background noise level was evaluated as the median of the absolute value of the acoustic samples over a 1-s window before being downsampled at $1 \mathrm{~Hz}$. This simple procedure is robust to the presence of transient signals, in our case echolocation signals emitted by the tagged animal.

Eight independent acoustic localizations with low measurement error were available (at $7^{\prime} 40,10^{\prime} 40,10^{\prime} 44,29^{\prime} 21,29^{\prime} 22,29^{\prime} 23,29^{\prime} 24$ and 29'33), obtained by cross-referencing data from AUTEC range hydrophones with the known times of emission of clicks from the tag [see Ward et al. (2011) for details]. These were ignored in the modelling, providing instead an independent comparison to our location results. For comparison, a conventional dead-reckoning track was obtained based on a state space model formulation with four states $(x, y, z$ and speed) and one observation (depth). Heading and pitch were treated as known covariates, fitted via a Kalman filter, implemented in R.

\section{Results}

The dive track reconstruction (for all three models) on a single MCMC chain would have required $65 \mathrm{~h}$ of computation time on a single core of a Intel ${ }^{\circledR}$ Xeon E5-2680v2 2.8Ghz 10-core processor. This was reduced to 75 min using HPR (Appendix S4).

Estimates of whale heading, pitch and roll for the complete dive are provided as Appendix S5. The standard deviations of the whale heading, pitch and roll estimates were $0.78^{\circ}$ (average for the whole dive, $95 \%$ in $\left.\left(0 \cdot 35^{\circ}, 1 \cdot 31^{\circ}\right)\right), 0 \cdot 35^{\circ}\left(0 \cdot 18^{\circ}, 0 \cdot 54^{\circ}\right)$, and $0.47^{\circ}\left(0 \cdot 14^{\circ}, 1 \cdot 01^{\circ}\right)$, respectively. These quantify observation measurement error in heading, pitch and roll. Animal speed is linearly predicted from log-transformed flow noise level $\left(R^{2}=0 \cdot 77\right.$, Appendix S2).

Deviance information criterion values are shown in Fig. 3. Model $\mathcal{M}_{0}$ was favoured from $1^{\prime}$ to $5^{\prime}$. Model $\mathcal{M}_{5}$ performed better for the rest of the dive except for 4 dive portions (at 12', $18^{\prime}, 25^{\prime}$ and $45^{\prime}$ ) where $\mathcal{M}_{10}$ was favoured. $\mathcal{M}_{0}$ better performance at the beginning of the dive (similar fit with lower complexity) can be explained by the whale's negligible pitch anomaly at this stage leading to the equal pitch assumption. The improvement provided by $\mathcal{M}_{5}$ and $\mathcal{M}_{10}$ for the rest of the dive (better fit despite higher complexity) suggests a non-negligible pitch anomaly and consequent need for eqn (6). Model $\mathcal{M}_{5}$ performed better than $\mathcal{M}_{10}$ for most of the dive (similar goodness-of-fit with lower complexity) indicating that the flexibility introduced by setting $\sigma_{p}=5^{\circ}$ should be preferred to $\sigma_{p}=10^{\circ}$. Nonetheless, $\mathcal{M}_{10}$ outperformed $\mathcal{M}_{5}$ for some dive portions (better fit despite higher complexity) with higher amplitude pitch anomaly. Overall, results strongly favour the unequal pitch assumption and $\sigma_{p}=5^{\circ}$. The following results are exclusively based on model $\mathcal{M}_{5}$, but this choice is not critical, as localization results are similar by using $\sigma_{p}=10^{\circ}$ (distance between tracks: $17.4 \pm 14.5 \mathrm{~m}$ ). The whale's estimated $3 \mathrm{D}$ track is illustrated in Fig. 4 (interval estimates are provided as Appendix S5). The absolute distance between the results from the independent acoustic survey localizations and the estimated track from $\mathcal{M}_{5}$ is $38.3 \pm 18.7 \mathrm{~m}$. For comparison, a standard dead-reckoning track fitted using a Kalman filter is also shown (distance between tracks: $151.6 \pm 88.9 \mathrm{~m}$ ). Estimated speed and pitch anomaly are illustrated in Fig. 5. The whale initiated its dive with a strongly negative pitch anomaly $\left(-20^{\circ}\right)$, pitch anomaly rapidly reached zero $\left(t \in\left[0^{\prime} 00,0^{\prime} 40\right]\right)$ and stabilized (peak-to-peak lesser than $4^{\circ}, t \in\left[2^{\prime} 00,6^{\prime} 00\right]$ and

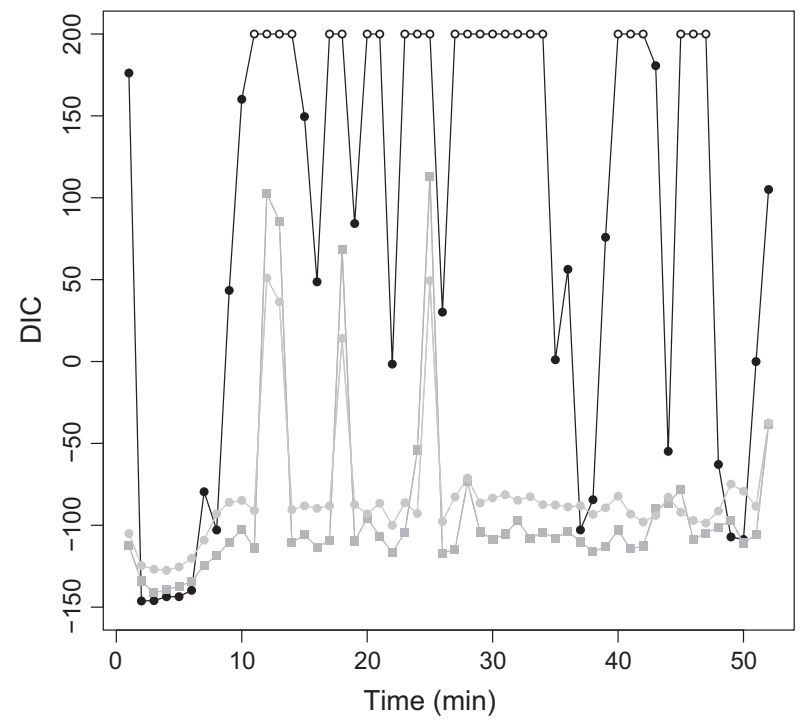

Fig. 3. DIC values computed separately for each minute of the dive for models $\mathcal{M}_{0}$ (black dots, values $>200$ are represented as empty dots), $\mathcal{M}_{5}$ (dark grey squares) and $\mathcal{M}_{10}$ (light grey circles). 


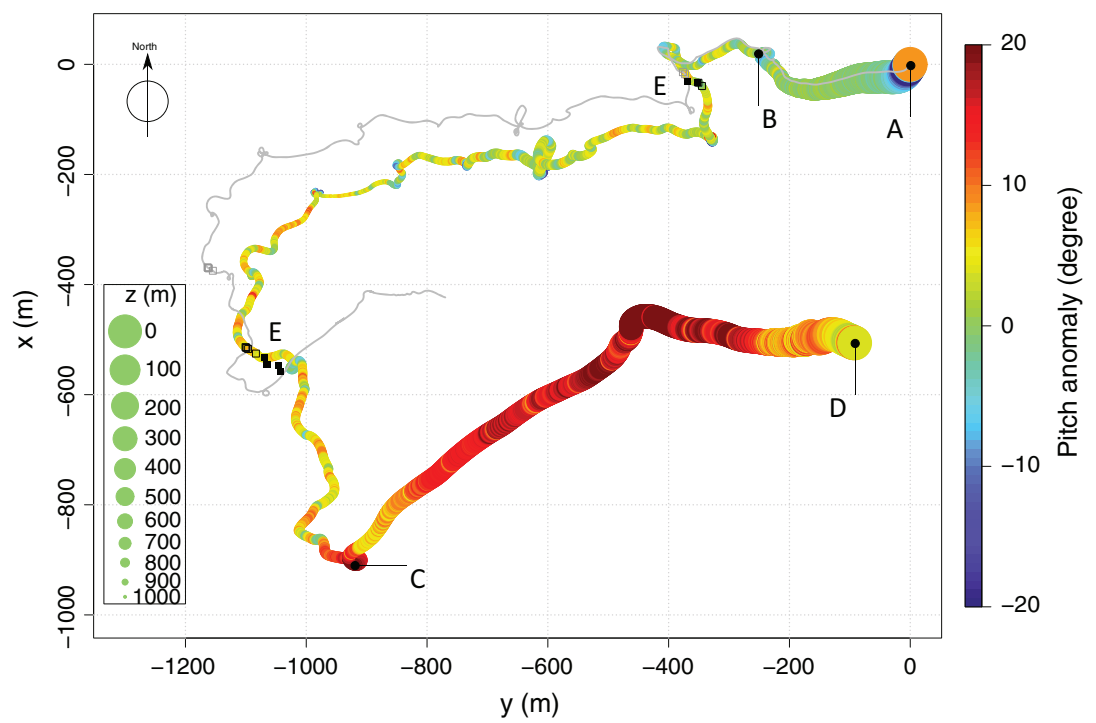

Fig. 4. Estimated 3D whale track ( $x$-axis, $y$ axis, dot size) and pitch anomaly (colour). The whale dives at $t_{0}=0(\mathrm{~A})$, ends its descent and starts to actively search for prey at depth at $t_{B}=7^{\prime} 50(\mathrm{~B})$, starts to reascend at $t_{C}=35^{\prime} 30$ (C), and resurfaces at $t_{\text {end }}=51^{\prime} 20$ (D). Independent acoustic localization from surrounding AUTEC hydrophones are represented (full black squares, E) together with points on the estimated track at the same timing (empty black squares). The whale covers a total curvilinear distance of $5170 \mathrm{~m}$ (descent (AB): $895 \mathrm{~m}$; at depth (BC): $2845 \mathrm{~m}$; ascent (CD): $1430 \mathrm{~m}$ ). Estimated whale track by processing accelerometer, magnetometer and depthmeter data with a Kalman filter is represented (grey line) together with location at acoustic localization timing (grey squares).

up to $15^{\circ}$ for $\left.t \in\left[6^{\prime} 00,7^{\prime} 50\right]\right)$. At depth $\left(t \in\left[7^{\prime} 50,35^{\prime} 30\right]\right)$, the whale alternated sections with either moderate pitch anomaly variations (peak-to-peak lesser than $10^{\circ}$ ) or strong variations (peak-to-peak up to $\left.40^{\circ}\right)$. During the ascent $\left(t \in\left[35^{\prime} 30,51^{\prime} 20\right]\right)$, the whale had a positive pitch anomaly (between $5^{\circ}$ and up to $28^{\circ}$ ). At depth, sections of large speed were associated with moderate pitch anomaly variations and sections of low speed were associated with strong pitch anomaly variations, suggesting that the whale alternated complex rotational movements at low speed and more regular movements at higher speed. During the ascent, the whale always kept a positive pitch, while the vertical speed could be negative (as low as $-0.40 \mathrm{~m} \mathrm{~s}^{-1}$ ) as illustrated in Fig. S2.2 (Appendix S2). The whale alternated active fluking (strong variations in speed) and passive gliding (no variation) with a strong positive pitch anomaly for the whole ascent.

\section{Discussion}

We used a relatively simple 'data-driven' model, where expected orientation is a function of accelerometer and magnetometer measurements, expected speed is a function of measured noise, and pitch anomaly is a function of speed and measured changed in depth. Measurement error on the observed quantities was assumed Gaussian, with known variance (except for variance in the speed vs. flow noise relationship, which was estimated). This approach can be expected to produce a realistic track where high-quality (i.e. low error), high-frequency data are available that relate closely to animal orientation and speed. DTAGs generate exactly such data. By contrast, where the data give less accurate information about animal movement or position, and/or are collected much less frequently, then it becomes necessary to include assumptions about the underlying movement behaviour of the animal in the model - for example using a biased correlated random walk, with model parameters representing centres of attraction or repulsion and correlation between time steps (McClintock et al. 2012). A good example of such data is Argos satellite tags, (McClintock et al. 2015). One advantage of our approach is that the track is not constrained by assumptions about movement behaviour. Disadvantages include it (i) requires high-quality data; (ii) does not incorporate biological knowledge of animal movement behaviour (except in the specification of different error variances in different diving phases); (iii) does not directly allow biological inferences about movement in contrast with, for example, the multistate models of McClintock et al. (2012) - although such inferences could be made in a second analysis stage; and (iv) cannot be used for simulating tracks, since it relies on input data at each time step. Therefore, the most appropriate approach depends on the data available and the goals of the analysis.

Reconstructing 3D tracks from accelerometer, magnetometer and depthmeter data alone, by implicitly assuming that the animal is moving in the direction of its longitudinal axis, might lead to biased inferences (see Fig. 4). As illustrated in Fig. S2.2 (Appendix S2), the whale's movement direction does not necessarily coincide with its longitudinal axis during the ascent. Therefore, the animal is capable of having a movement direction different to its own axis, issuing a serious warning against the equal pitch assumption. The inability to estimate speed when the animal is approximately horizontal (Appendix S2) represents an additional argument against reconstructing $3 \mathrm{D}$ tracks from accelerometer, magnetometer and depthmeter data alone.

Following previous work (Simon et al. 2009; Ware, Friedlaender \& Nowacek 2011), we estimated speed from an independent source, modelling the speed/noise relationship using the animal's steep descent phase, formalized via a loglinear relationship. The estimated track consistency with independent acoustic locations suggests that this procedure is sensible, at least for the first $30 \mathrm{~min}$ of the dive when acoustic data were available. However, using flow noise as a proxy for animal speed has its own limitations. It can be sensitive to changes in background noise during the dive (e.g. presence of sonar, boat motor, animal sounds). Difficulties are expected if the goal is to reconstruct tracks at the surface, when other sources might contribute significantly to acoustic noise (e.g. wave lapping) a solution for this is discussed later. Further, animal speed 

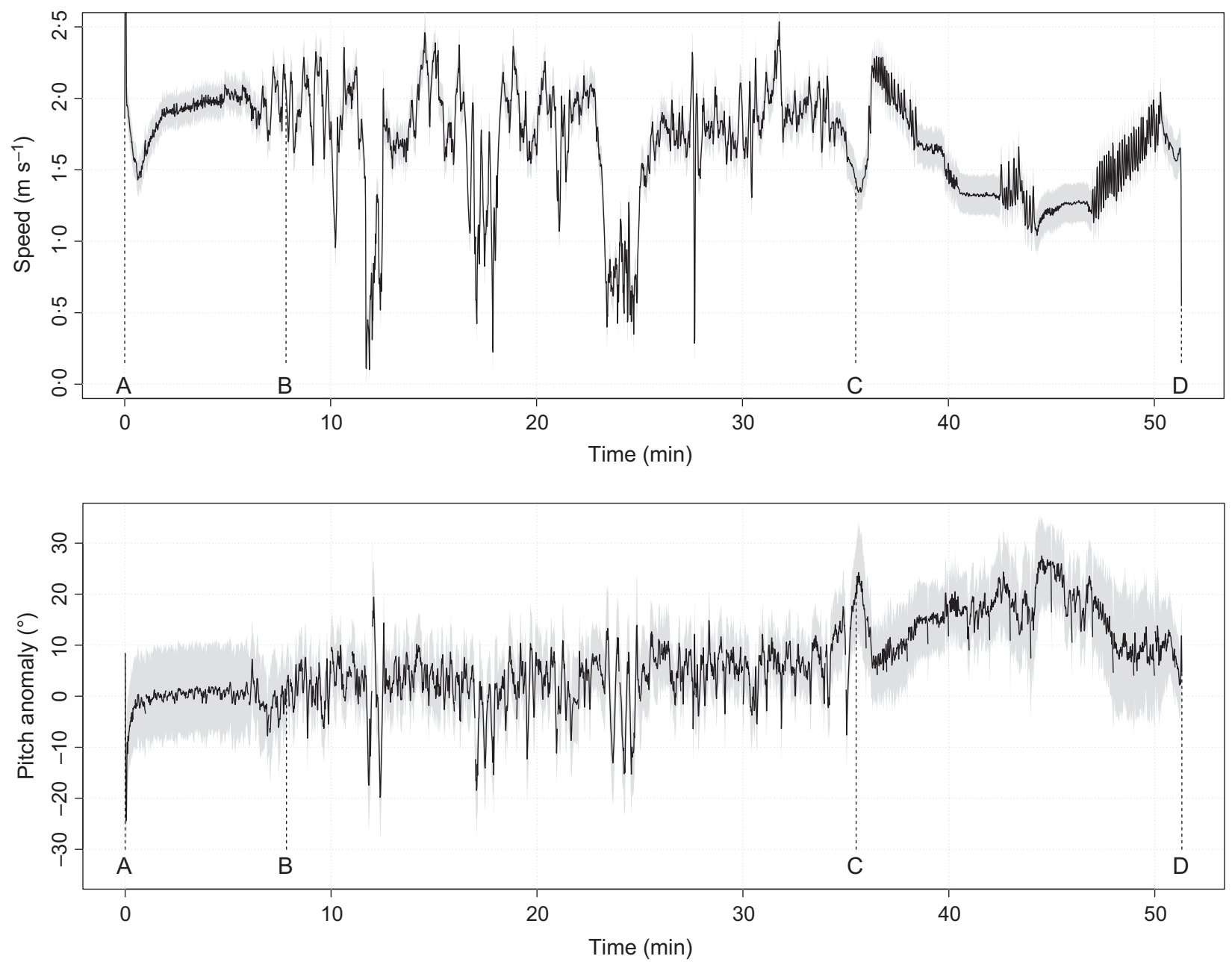

Fig. 5. Point estimate of whale speed (top, in black) and pitch anomaly (bottom, in black). Descent (AB), at depth (BC) and ascent (CD) phases are defined in Fig. 4. Mean speed during the descent is $1.91 \pm 0.17 \mathrm{~m} \mathrm{~s}^{-1}, 1.72 \pm 0.42 \mathrm{~m} \mathrm{~s}^{-1}$ at depth, and $1.51 \pm 0.28 \mathrm{~m} \mathrm{~s}^{-1}$ during the ascent. Mean pitch anomaly is $-0.5 \pm 2.9^{\circ}$ during the descent, $3.5 \pm 5.6^{\circ}$ at depth and $14.8 \pm 5.5^{\circ}$ during the ascent. Interval estimates are also represented on the plots (in grey). At depth, sections of large speed are associated with small pitch anomaly variations, and vice versa.

estimates from flow noise assume that the speed-flow noise relationship is independent of the animal orientation (discussed in more detail later).

The key advantage of including an independent estimate of speed was the ability to relax the equal pitch assumption, clearly supported by the data (Fig. S2.2) and by our localization results. For example, the whale was able to be oriented upwards while moving downwards (e.g. during the ascent), with differences up to $28^{\circ}$ between $3 \mathrm{D}$ orientation of its longitudinal axis and its speed vector. Consequently, accounting for complex animal movements by dissociating animal translation and rotation movements seems necessary to produce reliable $3 \mathrm{D}$ tracks. We have considered a fixed, known variance for pitch anomaly and concluded that a $5^{\circ}$ was a sensible choice for our example. Another approach might be to consider an unknown variance for pitch anomaly. Hence, provided a reasonable vague prior, variance would be estimated while reconstructing the track, and (at least in theory) a time-dependent variance might be considered.
We considered DIC as a model selection metric because it was readily implemented in OpenBUGS. We acknowledge DIC's use is controversial and that other approaches have been suggested [see, e.g., discussion papers following Spiegelhalter et al. (2002, 2014)]. It may, for example, be possible to implement a Gibbs variable selection or related approach [see O'Hara \& Sillanpää (2009) for review] to estimate the posterior model probability for a model with 0 variance in pitch anomaly vs a model with a non-zero variance prior.

Pitch anomaly does not necessarily describe a pitch movement of the animal in its own frame; instead, it is the difference between the animal's longitudinal axis pitch and the pitch of its speed vector (both on the Earth frame). Depending on the animal's roll, pitch anomaly can be the result of a pitch movement (in the animal frame) if roll is null or equal to $\pm 180^{\circ}$, of a heading movement (in the animal frame) if roll is equal to $\pm 90^{\circ}$, or a combination of both. Average roll was $4.9^{\circ}(95 \%$ in $\left.\left(-39 \cdot 6^{\circ}, 20 \cdot 5^{\circ}\right)\right)$ during the descent, $-5 \cdot 0^{\circ}\left(-53 \cdot 7^{\circ}, 35 \cdot 2^{\circ}\right)$ at depth, and $1.0^{\circ}\left(-15 \cdot 8^{\circ}, 23 \cdot 0^{\circ}\right)$ during the ascent. Consequently, variations in pitch anomaly here mainly depict pitch 
movements (in the animal frame) slightly combined with heading movements. We have not included heading anomaly in the model. Similarly as for pitch, heading anomaly could be defined as the difference between the heading of the longitudinal axis of the animal and the heading of its speed vector. A positive heading anomaly would represent movements when the animal points its longitudinal axis more on the starboard side than expected by its swimming direction, and vice versa. The reason for not including heading anomaly in the model is that it is not possible, given the available data, to compute both pitch and heading anomalies. Considering only pitch anomaly is a parsimonious choice: the most likely explanation for the discrepancy between measured depth and the depth predicted by the $3 \mathrm{D}$ orientation of the animal and its speed norm is through a vertical shift of the speed vector, that is pitch anomaly.

The model handles four sources of errors: observation measurement errors on accelerometer/magnetometer data $\left(\Sigma_{A}\right.$ and $\left.\Sigma_{M}\right)$, on depth data $\left(\sigma_{z}^{2}\right)$ and internal errors due to differences between $3 \mathrm{D}$ orientations of the animal body and speed $\left(\sigma_{p}^{2}\right)$, and on the prediction of speed from flow noise $\left(\sigma_{v}^{2}\right)$. The model propagates measurement and process errors into parameter estimate errors. However, it still apparently underestimates the location estimates precision, as indicated by the independent acoustic localizations (Fig. 4 and Appendix S5). Variances of parameter estimates are conditional on the model being true. This is strictly unrealistic, as the model still represents an oversimplification of the mechanism underlying animal 3D displacement and flow noise. Therefore, while ignoring them should be avoided, confidence intervals associated with locations should be handled with caution.

There are (at least) four additional sources of errors ignored by the model: (i) Strictly, the speed considered is the speed of the animal with respect to the water mass. We consequently reconstructed the track in the water mass frame, not in the Earth frame. If water speed (in the Earth frame) is not negligible with respect to animal speed (in the water mass frame), track reconstruction might be biased. Were current speeds available, one could incorporate them by adding a correction term in eqn (8); (ii) the calibration of the orientation of the tag to the whale frame was assumed to be an error-free process, and potential tag shift over time ignored. An option would be to estimate calibration angles while reconstructing the track to propagate calibration errors to uncertainties on animal 3D orientation. Further research on the impacts of this calibration procedure on DTAG based by-products is welcome; (iii) while errors on the prediction of the speed from the noise level are considered (eqn 7), errors on the parameters of the relationship $\left(a_{v}, b_{v}, \sigma_{v}\right)$ or on the relationship itself are ignored - the use of a more advanced relationship, calibrated while reconstructing the track is an interesting perspective; (iv) a known error-free variance $\sigma_{p}^{2}$ was used. As mentioned earlier, an option would be to estimate $\sigma_{p}^{2}$. The consequences of assuming a known calibrated speed-noise relationship and a known variance $\sigma_{p}^{2}$ on the track reconstruction process are explored in Appendix S6.
No explicit track smoothing was implemented. The reconstructed track regularity (Fig. 4) is the consequence of the estimated speed regularity (Fig. 5), itself the consequence of flow noise regularity, caused by smooth animal movement. Another option to smooth the track would be to consider explicitly autocorrelation in animal 3D orientation and speed. This might help when speed could not be inferred from flow noise (e.g. tags without acoustic sensors). One possible implementation is to add two sets of latent variables, angular speeds $\left(v_{h}(t)\right.$, $v_{p}(t), v_{r}(t)$, e.g. $\left.v_{h}(t)=(h(t+\Delta t)-h(t)) / \Delta t\right)$ and accelerations $\left(a_{x}(t), a_{y}(t), a_{z}(t)\right.$, e.g. $\left.a_{x}(t)=\left(v_{x}(t+\Delta t)-v_{x}(t)\right) / \Delta t\right)$, assumed unbiased with known behavioural state-dependent variances. As an illustration, the angular speed statistics (mean \pm standard deviation) of our whale differ across behavioural states: descent (pitch: $-1.0 \pm 3.7^{\circ} \mathrm{s}^{-1}$; heading: $0.0 \pm 2.0^{\circ}$ $\mathrm{s}^{-1}$; roll: $\left.0.5 \pm 3.0^{\circ} \mathrm{s}^{-1}\right)$, at depth $\left(-0.8 \pm 5.5^{\circ} \mathrm{s}^{-1} ;-0.1 \pm\right.$ $\left.5.0^{\circ} \mathrm{s}^{-1} ; 0 \cdot 0 \pm 5 \cdot 0^{\circ} \mathrm{s}^{-1}\right)$ and ascent $\left(-0.2 \pm 3.0^{\circ} \mathrm{s}^{-1} ; 0.0 \pm\right.$ $2.5^{\circ} \mathrm{s}^{-1} ; \quad 0.0 \pm 2.2^{\circ} \mathrm{s}^{-1}$ ). Acceleration (three coordinates altogether) also differs across states: descent $(0.000 \pm$ $\left.0.091 \mathrm{~m} \mathrm{~s}^{-2}\right)$, at depth $\left(0.001 \pm 0.200 \mathrm{~m} \mathrm{~s}^{-2}\right)$ and ascent $\left(0.000 \pm 0.081 \mathrm{~m} \mathrm{~s}^{-2}\right)$. The latter values could also be used to smooth animal tracks computed from acoustic surveys, as described by Laplanche (2012).

One of the advantages of implementing the model in a Bayesian framework is that incorporation of additional data sources and propagating corresponding observation errors is conceptually straightforward. Acoustic-based localization could be used as direct observations or provide time of arrival differences (TDOA) data instead of computed localization, by combining our model with that of Laplanche (2012), which would deal with propagating TDOA errors to localization estimates.

We made some approximations to speed up model fitting computations: (i) we broke the full model into three parts (3D orientation, speed-flow noise and track reconstruction) and (ii) analysed some parts in 1-min chunks, using Gaussian distributions to cascade uncertainty between chunks (see Section 'Bayesian computation and approximating model' and Appendix S4). These approximations are expected to have a negligible influence on the estimated track since they concern only the variance of orientation and position. Nevertheless, we see four main drawbacks in our implementation: (i) it is not compatible with additional independent positional information (GPS or acoustic based), except for at the first time point; (ii) it removes the possibility to correct for animal acceleration while computing animal orientation from accelerometer data. Although animal acceleration is negligible for large species, such as the beaked whale considered here, it would be questionable for smaller, rapid species such as dolphins or pinnipeds; (iii) it prevents calibrating tag orientation while reconstructing the track; and (iv) it removes the possibility to account for animal orientation and speed to predict flow noise and compare to data for the whole dive.

Clearly, HPR are a valuable tool, giving the potential to speed up extensive computations. Whether this potential is realized is case specific: in our case, because of the 
independence of some latent variables over time, parts of the computation could be carried out in parallel with almost no loss in inference accuracy. This might no longer be the case if the model were extended. Another option to reduce computation time might be implementing the model in a likelihoodbased approach, for example via an extended Kalman filter, another research avenue we are pursuing.

Reconstructing tracks from accelerometer, magnetometer and depthmeter tag data happens routinely regardless of potential hidden dangers in doing so. The need for methods incorporating observation error and providing precision measures on estimated tracks is clear. We have shown that the approach described here, allowing (i) the estimation of speed from flow noise and consequently (ii) the dissociation of the $3 \mathrm{D}$ orientation of the animal longitudinal axis and the $3 \mathrm{D}$ orientation of its speed vector, is an important step towards such goal. We suggest that practitioners should evaluate the validity of the equal pitch assumption on their species before reconstructing 3D tracks. Our methods - considering equal/unequal pitch assumption, comparing outputs and fits, and using independent localization - are an option. It allowed us to design a new descriptor on marine mammal movement: pitch anomaly. We believe that making assumptions explicit via a mathematical model is a relevant approach in gathering current knowledge about animal behaviour, identifying gaps and allowing new insights.

\section{Acknowledgements}

Access to the HPC resources of CALMIP was granted under the allocation 2014P1421. TAM was funded under grant number N000141010382 from the Office of Naval Research (LATTE project). A number of extremely useful discussions with Mark Johnson provided insights on various aspects of this analysis. Points of view that are expressed in the present work are not to be taken to reflect the views of MJ. Jessica Ward provided comments on earlier versions of the manuscript, helped gathering the beaked whale data and provided AUTEC's independent acoustic localizations. Stacy DeRuiter provided comments and encouragement throughout. Tag data were facilitated by Peter L. Tyack. Tagging performed under US National Marine Fisheries Service research permit numbers 981-157802 and 981-1707-00 to PLT and with the approval of the Woods Hole Oceanographic Institution Animal Care and Use Committee. We acknowledge the considerable improvements to the paper thanks to suggestions from the reviewing process.

\section{Data accessibility}

The DTAG data used to illustrate the methods are available from the Dryad Digital Repository: http://dx.doi.org/10.5061/dryad.138cg (Laplanche, Marques \& Thomas 2015).

\section{References}

Battaile, B. (2014) Track Reconstruction: Reconstruct Animal Tracks from Magnetometer, Accelerometer, Depth and Optional Speed Data. R package version 1.1. URL http://CRAN.R-project.org/package $=$ TrackReconstruct ion [accessed 02 April 2014].

Beyer, H.L., Morales, J.M., Murray, D. \& Fortin, M.J. (2013) The effectiveness of Bayesian state-space models for estimating behavioural states from movement paths. Methods in Ecology and Evolution, 4, 433-441.

Bograd, S.J., Block, B.A., Costa, D.P. \& Godley, B.J. (2010) Biologging technologies: new tools for conservation. Introduction. Endangered Species Research, 10, 1-7.

Burgess, W.C. (2009) The Acousonde: a miniature autonomous wideband recorder. The Journal of the Acoustical Society of America, 125, 2588-2588.
Davis, R.W., Fuiman, L.A., Williams, T.M. \& Boeuf, B.J.L. (2001) Three-dimensional movements and swimming activity of a northern elephant sea. Comparative Biochemistry and Physiology Part A, 129, 759-770.

Farrell, E. \& Fuiman, L. (2013) AnimalTrack: Animal Track Reconstruction for High Frequency 2-dimensional (2D) or 3-dimensional (3D) Movement Data. $\mathrm{R}$ package version 1.0.0. URL http://CRAN.R-project.org/package $=$ animalTrack [accessed 02 April 2014].

Friedlaender, A.S., Hazen, E.L., Nowacek, D.P., Halpin, P.N., Ware, C., Weinrich, M.T., Hurst, T. \& Wiley, D. (2009) Diel changes in humpback whale Megaptera novaeangliae feeding behavior in response to sand lance Ammodytes spp. behavior and distribution. Marine Ecology Progress Series, 395, 91100.

Gelman, A., Carlin, J.B., Stern, H.S. \& Rubin, D.B. (2003) Bayesian Data Analysis. Chapman and Hall/CRC, Boca Raton.

Hazen, E.L., Friedlaender, A.S., Thompson, M.A., Ware, C.R., Weinrich, M.T., Halpin, P.N. \& Wiley, D.N. (2009) Fine-scale prey aggregations and foraging ecology of humpback whales Megaptera novaeangliae. Marine Ecology Progress Series, 395, 75-89.

International Association of Geomagnetism and Aeronomy, Working Group VMOD (2010) International geomagnetic reference field: the eleventh generation. Geophysical Journal International, 183, 1216-1230.

Johnson, M.P. \& Tyack, P.L. (2003) A digital acoustic recording tag for measuring the response of wild marine mammals to sound. IEEE Journal of Oceanic Engineering, 28, 3-12.

Johnson, M., Aguilar de Soto, N. \& Madsen, P.T. (2009) Studying the behaviour and sensory ecology of marine mammals using acoustic recording tags: a review. Marine Ecology Progress Series, 395, 55-73.

Jonsen, I.D., Flemming, J.M. \& Myers, R.A. (2005) Robust state-space modeling of animal movement data. Ecology, 86, 2874-2880.

Jonsen, I., Basson, M., Bestley, S., Bravington, M., Patterson, T., Pedersen, M., Thomson, R., Thygesen, U. \& Wotherspoon, S. (2012) State-space models for bio-loggers: a methodological road map. Deep Sea Research Part II: Topical Studies in Oceanography. 88-89, 34-46.

Langrock, R., Marques, T.A., Thomas, L. \& Baird, R.W. (2013) Modeling the diving behavior of whales: a latent-variable approach with feedback and semiMarkovian components. Journal of Agricultural, Biological, and Environmental Statistics, 19, 82-100.

Langrock, R., Hopcraft, J.G.C., Blackwell, P.G., Goodall, V., King, R., Niu, M. et al. (2014) A model for group dynamic animal movement. Methods in Ecology \& Evolution, 5, 190-199.

Laplanche, C. (2012) Bayesian three-dimensional reconstruction of toothed whale trajectories: Passive acoustics assisted with visual and tagging measurements. The Journal of the Acoustical Society of America, 132, 3225-3233.

Laplanche, C., Marques, T. \& Thomas, L. (2015) Data from: Tracking marine mammals in $3 D$ using electronic tag data. Dryad Digital Repository, http:// dx.doi.org/10.5061/dryad.138cg.

Madsen, P.T., Johnson, M., de Soto, N.A., Zimmer, W.M.X. \& Tyack, P. (2005) Biosonar performance of foraging beaked whales (Mesoplodon densirostris). The Journal of Experimental Biology, 208, 181-194.

Marshall, G., Bakhtiari, M., Shepard, M., Tweedy III J., Rasch, D., Abernathy, K., Joliff, B., Carrier, J.C. \& Heithaus, M.R. (2007) An advanced solid-state animal-borne video and environmental data-logging device ("Crittercam") for marine research. Marine Technology Society Journal, 41, 31-38.

McClintock, B.T., King, R., Thomas, L., Matthiopoulos, J., McConnell, B.J. \& Morales, J.M. (2012) A general modeling framework for animal movement and migration using multi-state random walks. Ecological Monographs, 82, 335-349.

McClintock, B., London, J., Cameron, M. \& Boveng, P. (2015) Modelling animal movement using the Argos satellite telemetry location error ellipse. Methods in Ecology and Evolution, 6, 266-277.

Mitani, Y., Sato, K., Ito, S., Cameron, M.F., Siniff, D.B. \& Naito, Y. (2003) A method for reconstructing three-dimensional dive profiles of marine mammals using geomagnetic intensity data: results from two lactating Weddell seals. Polar Biology, 26, 311-317.

Morales, J.M., Haydon, D.T., Frair, J., Holsinger, K.E. \& Fryxell, J.M. (2004) Extracting more out of relocation data: building movement models as mixtures of random walks. Ecology, 85, 2436-2445.

Ntzoufras, I. (2009) Bayesian Modeling Using WinBUGS. Wiley series in computational statistics.John Wiley \& Sons, Inc., Hoboken, NJ.

O’Hara, R. \& Sillanpää, M. (2009) A review of Bayesian variable selection methods: what, how and which. Bayesian Analysis, 4, 85-118.

R Core Team (2013) R: A Language and Environment for Statistical Computing. R Foundation for Statistical Computing, Vienna, Austria. 
Ropert-Coudert, Y. \& Wilson, R.P. (2005) Trends and perspectives in animalattached remote sensing. Frontiers in Ecology and the Environment, 3, 437-444.

Rutz, C. \& Troscianko, J. (2013) Programmable, miniature video-loggers for deployment on wild birds and other wildlife. Methods in Ecology and Evolution, 4, 114-122.

Shaffer, J.W., Moretti, D., Jarvis, S., Tyack, P. \& Johnson, M. (2013) Effective beam pattern of the Blainville's beaked whale (Mesoplodon densirostris) and implications for passive acoustic monitoring. The Journal of the Acoustical Society of America, 133, 1770-1784.

Shiomi, K., Sato, K., Mitamura, H., Arai, N., Naito, Y. \& Ponganis, P.J. (2008) Effect of ocean current on the dead-reckoning estimation of 3-D dive paths of emperor penguins. Aquatic Biology, 3, 265-270.

Simon, M., Johnson, M., Tyack, P. \& Madsen, P.T. (2009) Behaviour and kinematics of continuous ram filtration in bowhead whales (Balaena mysticetus). Proceedings of the Royal Society B: Biological Sciences, 276, 3819-828.

Simon, M., Johnson, M. \& Madsen, P.T. (2012) Keeping momentum with a mouthful of water: behavior and kinematics of humpback whale lunge feeding. The Journal of Experimental Biology, 215, 3786-3798.

Spiegelhalter, D., Best, N., Carlin, B. \& van der Linde, A. (2002) Bayesian measures of model complexity and fit. Journal of the Royal Statistical Society: Series B (Statistical Methodology), 64, 583-639.

Spiegelhalter, D., Best, N., Carlin, B. \& van der Linde, A. (2014) The deviance information criterion: 12 years on. Journal of the Royal Statistical Society: Series B (Statistical Methodology), 76, 485-493.

Tracey, J.A., Sheppard, J., Zhu, J., Wei, F., Swaisgood, R.R. \& Fisher, R.N. (2014) Movement-based estimation and visualization of space use in 3D for wildlife ecology and conservation. PLoS One, 9, e101205.

Tyson, R.B., Friedlaender, A.S., Ware, C., Stimpert, A.K. \& Nowacek, D.P. (2012) Synchronous mother and calf foraging behaviour in humpback whales Megaptera novaeangliae: insights from multi-sensor suction cup tags. Marine Ecology Progress Series, 457, 209-220.

Ward, J., Jarvis, S., Moretti, D., Morrissey, R., DiMarzio, N., Thomas, L. \& Marques, T.A. (2011) Beaked whale (Mesoplodon densirostris) passive acoustic detection with increasing ambient noise. The Journal of the Acoustical Society of America, 129, 662-669.

Ware, C., Arsenault, R., Plumlee, M. \& Wiley, D. (2006) Visualizing the underwater behavior of humpback whales. IEEE Computer Graphics and Applications, 26, 14-18.

Ware, C., Friedlaender, A.S. \& Nowacek, D.P. (2011) Shallow and deep lunge feeding of humpback whales in fjords of the West Antarctic Peninsula. Marine Mammal Science, 27, 587-605.

Ware, C., Wiley, D.N., Friedlaender, A.S., Weinrich, M., Hazen, E.L., Bocconcelli, A. et al. (2014) Bottom side-roll feeding by humpback whales (Megaptera novaeangliae) in the southern Gulf of Maine, U.S.A. Marine Mammal Science, 30, 494-511.

Watwood, S.L., Miller, P.J.O., Johnson, M., Madsen, P.T. \& Tyack, P.L. (2006) Deep-diving foraging behaviour of sperm whales (Physeter macrocephalus). Journal of Animal Ecology, 75, 814-825.

Wilson, R.P., Liebsch, N., Davies, I.M., Quintana, F., Weimerskirch, H., Storch, S. et al. (2007) All at sea with animal tracks; methodological and analytical solutions for the resolution of movement. Deep Sea Research Part II: Topical Studies in Oceanography, 54, 193-210.

Zimmer, W.M.X., Tyack, P.L., Johnson, M.P. \& Madsen, P.T. (2005) Three-dimensional beam pattern of regular sperm whale clicks confirms bent-horn hypothesis. The Journal of the Acoustical Society of America, 117, 1473-1485.

Received 27 January 2015; accepted 5 March 2015

Handling Editor: Olivier Gimenez

\section{Supporting Information}

Additional Supporting Information may be found in the online version of this article.

Appendix S1. Statistical model for accelerometer and magnetometer measurement errors.

Appendix S2. Statistical model for speed from background noise level.

\section{Appendix S3. BUGS code.}

Appendix S4. Procedure to distribute track computations on a High Performance Resource (HPR).

Appendix S5. Point and interval estimates of the heading, pitch, roll, and coordinates of the whale for the complete dive.

Appendix S6. Investigating sensitivity to variance in pitch anomaly and flow noise relationship. 